\title{
A Rapidly Progressive Variant of Psoriatic Arthritis: Psoriatic Onycho-Pachydermo-Periostitis
}

\author{
Sibel SÜZEN ÖZBAYRAK, ${ }^{1}$ Vildan BİNAY SAFER, ${ }^{1}$ Mustafa ÖZBAYRAK ${ }^{2}$ \\ ${ }^{1}$ Department of Physical Medicine and Rehabilitation, Haydarpaşa Numune Training and Research Hospital, İstanbul, Turkey \\ ${ }^{2}$ Department of Radiology, İstanbul University Cerrahpaşa Medical Faculty Hospital, İstanbul, Turkey
}

\begin{abstract}
We present a case with psoriatic onychodystrophy, onycholysis, connective tissue thickening above the distal phalanx as well as specific radiologic changes diagnosed as psoriatic onycho-pachydermo-periostitis who responded dramatically to methotrexate with current literature review. In this article, we report a 53-year-old male patient with psoriatic onycho-pachydermo-periostitis who responded dramatically to methotrexate.

Keywords: Methotrexate; psoriatic arthritis; psoriatic onycho-pachydermo-periostitis; variant.
\end{abstract}

Psoriatic onycho-pachydermo-periostitis (POPP) was initially described by Fournie B in $1989 .{ }^{1}$ It is an uncommon subset of psoriatic arthritis that is characterized by psoriatic onychodystrophy, onycholysis, connective tissue thickening above the distal phalanx as well as specific radiologic changes such as periosteal reaction and bone erosions of the distal phalanges. ${ }^{1}$ In this article, the case of a 53-year-old male patient with POPP who responded dramatically to methotrexate has been discussed.

\section{CASE REPORT}

A 53-year-old male patient was admitted to the dermatology clinic with nail changes and pain in all the fingers, which started approximately one year prior to admission. His nail biopsies were unremarkable and his condition was unresponsive to anti-fungal medications ordered by several dermatology clinics over the past one year. Thereafter, the patient was referred to our clinic for flexion deformity of the distal interphalangeal (DIP) joints. He had painful swellings with functional impairment in the DIP joints for seven to eight months after which the deformities started to develop. A written informed consent was obtained from the patient.

Our patient was working as a documentalist with no history of trauma or repetitive injury. On rheumatologic evaluation, there were no remarkable findings such as recurrent genital aphthous ulcers, oral aphthous ulcers, dry eyes and mouth, back pain, morning stiffness, bowel related symptoms, repeated fever or abdominal pain, and any other dermatologic rash. He had no systemic diseases, history of infection, recent vaccinations, or regular drug use. Family history was negative for psoriasis or other skin diseases. A physical examination revealed erythematous swellings and flexion deformities in all the DIP joints. All the fingernails had severe dystrophic changes. A psoriatic plaque was present on the extensor surface of the right elbow. 
A complete blood count, electrolyte, and acute phase reactant tests revealed no abnormalities. Rheumatologic markers including the rheumatoid factor and anti-citrullinated antibody were all negative. Direct radiography revealed an evidence of intraarticular erosion, periosteal reaction, and prominent thickened periarticular and paraosseous soft tissues (Figure 1). A magnetic resonance imaging of the hands showed irregularities of the DIP joints in $T_{1}$ weighted images (Figure 2). Based on the findings mentioned above, the patient was diagnosed as having POPP. The patient was started on methylprednisolone $5 \mathrm{mg} /$ day and methotrexate $10 \mathrm{mg} /$ week, which increased to $15 \mathrm{mg} /$ week after two weeks. The patient was evaluated after four weeks of treatment and liver function tests; the creatinine and complete blood counts were normal and there was an 80\% improvement in the pain scores $(10 \mathrm{~cm}$ visual analog scale) for arthralgia. The patient was reevaluated after three months of treatment and there was a 90\% improvement in the pain scores compared to the scores at admission and new nail formations at the base of the nail beds were observed.

\section{DISCUSSION}

To the best of our knowledge, 22 cases of POPP have been reported in the literature. ${ }^{2}$ POPP is considered to be an uncommon subset of psoriatic arthritis that is characterized by psoriatic onychodystrophy, onycholysis, connective tissue thickening above the distal phalanx as well as specific radiologic changes such as periosteal reaction and bone erosions

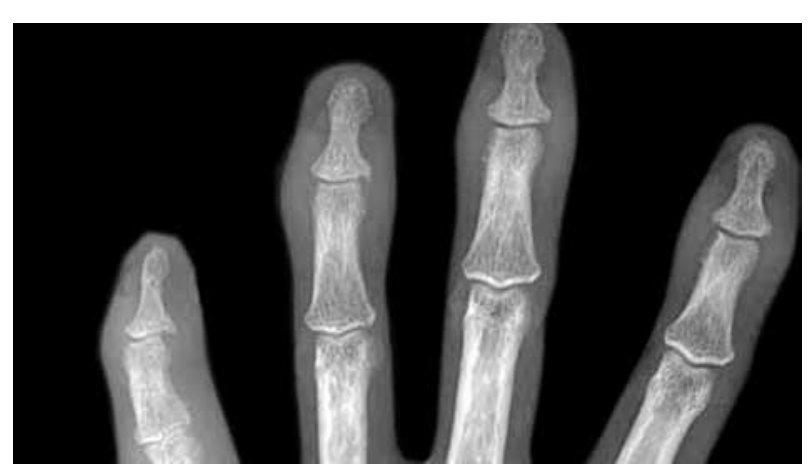

Figure 1. In close view of frontal radiograph of hand: Intraarticular erosion and periosteal reaction and prominent thickened periarticular soft tissues. of the distal phalanges. ${ }^{1}$ These changes in nail, bone, and connective tissue result in a drumsticklike deformity of the digits. The pathology and pathophysiology of POPP are poorly understood. A possible pathogenic mechanism could be inflammation commencing from subungual structures and spreading between the tendons and bones of the terminal phalanx. ${ }^{3}$ Patients usually have associated tenderness of the involved joints with functional impairment. POPP has been reported in various small joints in both upper and lower extremities, although the great toes are involved in most cases. ${ }^{4}$

Joint and nail involvement in psoriasis is relatively common. ${ }^{5}$ In contrast, bony involvement of the terminal phalanx under a psoriatic nail is rare. ${ }^{6}$ The approximate time of progress from the initiation of nail changes to the beginning of erosion or deformity in digits has not been reported in the literature except in one case where it was observed that psoriatic onychodystrophy and painful swellings of the toes and fingers developed within six months. ${ }^{7}$ In our case, painful swelling and tenderness of the fingers started three to four weeks after nail changes were observed and within three months

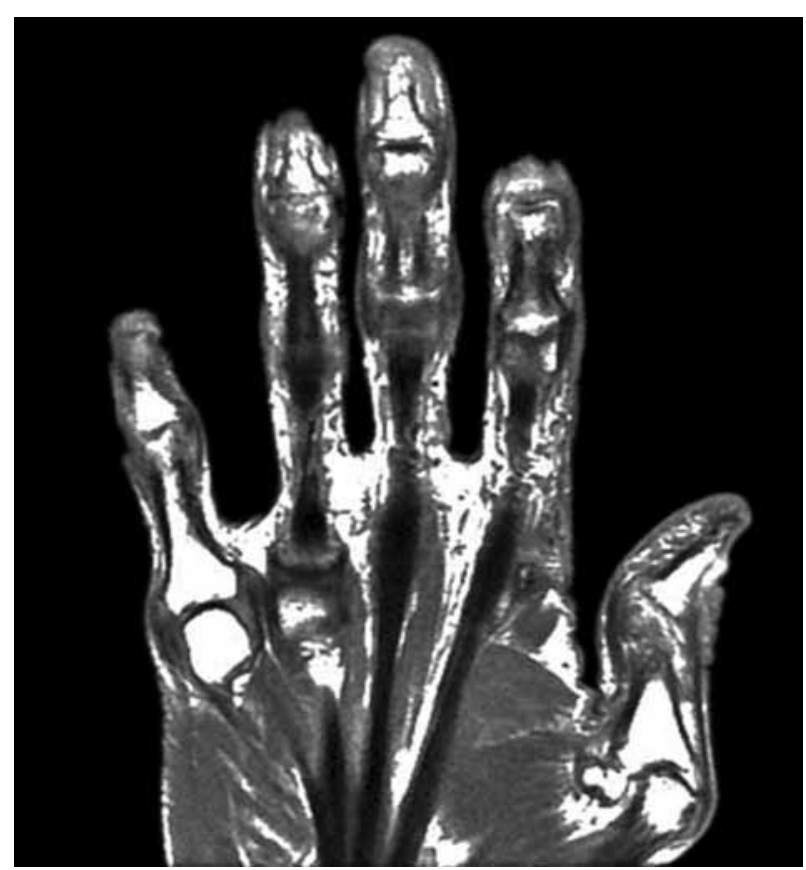

Figure 2. $T_{1}$ weighted magnetic resonance imaging of hand: Irregularities in margins of distal interphalangeal joints are seen. 
as of the development of the flexion deformities of the DIP joints.

Psoriatic onycho-pachydermo-periostitis is diagnosed by clinical and laboratory evaluation and radiological investigations such as direct radiography, ultrasonography, and magnetic resonance imaging. ${ }^{8}$ In our case, typical nail changes in both upper extremities were present. Although psoriasis had not been diagnosed previously, a scaly psoriatic plaque was present in the extensor aspect of the right elbow. Despite these findings, the patient was treated with anti-fungal medications for a long time as observed in most POPP cases cited in the literature.

Radiographic findings in POPP include intraand periarticular erosions, new bone formation including periostitis, bony protuberances, soft tissue prominence (dactylitis or sausage digits), and calcification at ligament and tendinous insertions in keeping with enthesopathy. ${ }^{9}$ Sanal et al. ${ }^{10}$ described a case of POPP with extensive bone marrow edema of the metacarpal bones without distinctive periostitis.

Treatment for POPP has not been standardized. Methotrexate $4 \mathrm{mg} /$ week was reportedly found to be effective in one patient. ${ }^{11}$ In another case, methotrexate and leflunomide combination therapy resulted in sufficient relief of symptoms. ${ }^{12}$ In one case, the nails began to grow regularly after six weeks of therapy with methotrexate (15 mg/week). ${ }^{7}$ In the literature, a female case with POPP was unresponsive to traditional systemic treatments and adalimumab, subsequently having a favorable response to treatment with etanercept. ${ }^{13}$ However, in another case, successful results were observed with adalimumab treatment. ${ }^{14}$ In our case, after a definite diagnosis was established, $10 \mathrm{mg} /$ week of methotrexate and $5 \mathrm{mg}$ of prednisolone was initiated. A week later, the dosage of methotrexate was increased to $15 \mathrm{mg} /$ week. After four weeks, the patient was re-evaluated and an $80 \%$ decrease in pain scores was detected. Decreases in pain scores were observed to continue three months later while new nail formations at the base of the nail beds were also noted.

In conclusion, owing to its rapid rate of progression, an accurate and early diagnosis of
POPP is crucial for initiating optimal therapy as soon as possible and preventing deformities.

\section{Declaration of conflicting interests}

The authors declared no conflicts of interest with respect to the authorship and/or publication of this article.

\section{Funding}

The authors received no financial support for the research and/or authorship of this article.

\section{REFERENCES}

1. Fournié B, Viraben R, Durroux R, Lassoued S, Gay R, Fournié A. Psoriatic onycho-pachydermoperiostitis of the big toe. Anatomo-clinical study and physiopathogenic approach apropos of 4 cases. Rev Rhum Mal Osteoartic 1989;56:579-82. [Abstract]

2. Mahoney JM, Scott R. Psoriatic onychopachydermoperiostitis (POPP): a perplexing case study. J Am Podiatr Med Assoc 2009;99:140-3.

3. McGonagle D, Stockwin L, Isaacs J, Emery P. An enthesitis based model for the pathogenesis of spondyloarthropathy. additive effects of microbial adjuvant and biomechanical factors at disease sites. $\mathrm{J}$ Rheumatol 2001;28:2155-9.

4. Kapusta MA, Dumont C. Differential response of psoriatic onycho-pachydermo-periostitis to 2 anti-tumor necrosis factor-alpha agents. J Rheumatol 2008;35:2077-80.

5. Cinar N, Bodur H, Eser F, Gul U, Gonul M, Deniz Oğuz I. The Prevalence and Characteristics of Psoriatic Arthritis in Patients With Psoriasis in a Tertiary Hospital. Arch Rheumatol 2015;30:23-7.

6. Boisseau-Garsaud AM, Beylot-Barry M, Doutre MS, Beylot C, Baran R. Psoriatic onycho-pachydermoperiostitis. A variant of psoriatic distal interphalangeal arthritis? Arch Dermatol 1996;132:176-80.

7. Kroiss MM, Vogt T, Finkenzeller T, Landthaler M, Stolz W. Psoriatic onycho-pachydermo- periostitis. Z Rheumatol 2002;61:598-600. [Abstract]

8. Matucci-Cerinic M, Lotti T, Calvieri S, Ghersetich I, Sacerdoti L, Teofoli $\mathrm{P}$, et al. The spectrum of dermatological symptoms of pachydermoperiostosis (primary hypertrophic osteoarthropathy): a genetic, cytogenetic and ultrastructural study. Clin Exp Rheumatol 1992;10:45-8.

9. Wollina U, Unger L, Heinig B, Kittner T. Psoriatic arthritis. Dermatol Ther 2010;23:123-36.

10. Sanal HT, Yilmaz S, Cinar M, Simsek I, Dinc A, Tayfun C. Onycho-pachydermatit with extensive bone marrow edema predominant in the metacarpals: a "forme fruste" of POPP? Rheumatol Int 2012;32:1449-52.

11. Ochiai T, Washio H, Shiraiwa H, Takei M, Sawada S. Psoriatic onycho-pachydermo-periostitis successfully treated with low-dose methotrexate. Med Sci Monit 2006;12:27-30. 
12. Romiti R, Santos D, Carvalho J, Arnone M, Takahashi MD. POPP syndrome: Psoriatic onychopachydermoperiostitis. Dermatol Online J 2013;19:6. [Abstract]

13. Dans M, Hivnor C, van Voorhees AS. Psoriatic onychopachydermoperiostitis: improvement with etanercept. Br J Dermatol 2005;153:858-9.

14. Bongartz T, Härle P, Friedrich S, Karrer S, Vogt T, Seitz A, et al. Successful treatment of psoriatic onychopachydermo periostitis (POPP) with adalimumab. Arthritis Rheum 2005;52:280-2. 